\title{
Analysis of Shane Telescope Aberration Before and After Collimation
}

Don Gavel

January 26, 1999

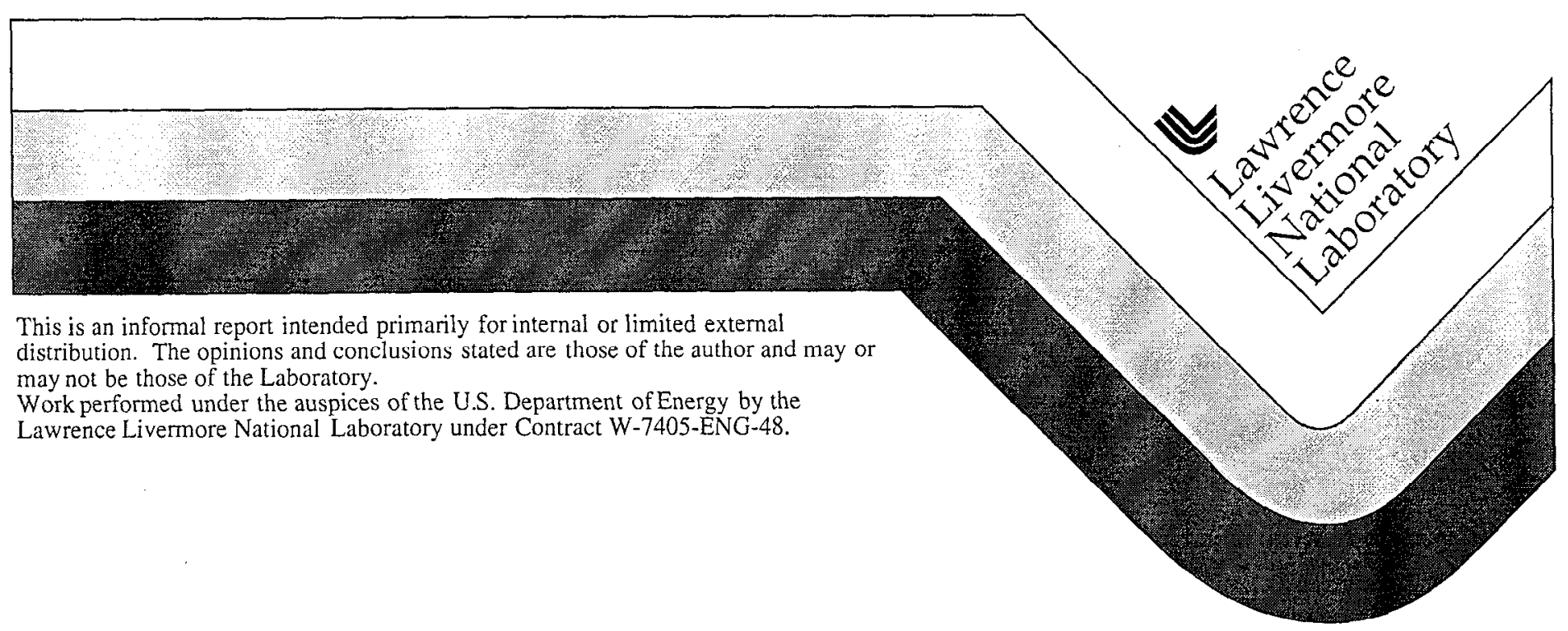




\section{DISCLAIMER}

This document was prepared as an account of work sponsored by an agency of the United States Government. Neither the United States Government nor the University of California nor any of their employees, makes any warranty, express or implied, or assumes any legal liability or responsibility for the accuracy, completeness, or usefulness of any information, apparatus, product, or process

disclosed, or represents that its use would not infringe privately owned rights. Reference herein to any specific commercial product, process, or service by trade name, trademark, manufacturer, or otherwise, does not necessarily constitute or imply its endorsement, recommendation, or favoring by the United States Government or the University of California. The views and opinions of authors expressed herein do not necessarily state or reflect those of the United States Government or the University of California, and shall not be used for advertising or product endorsement purposes. 


\title{
Analysis of Shane Telescope Aberration Before and After Collimation
}

\author{
Don Gavel, LLNL
}

\begin{abstract}
The Shane 3 meter telescope was recollimated in August 1998 to remove aberrations that were perceived to affect image quality in good seeing conditions. The Lick Adaptive Optics system is capable, indirectly, of measuring the static aberrations introduced by the telescope primary and secondary mirror pair. Since AO runs were scheduled both before and after the collimation, this allowed a comparison of the pre and post collimation telescope aberrations. The absolute calibration of the wavefront measurement is complicated by the indirect measurement technique, which uses the shape of the deformable mirror in its closed-loop corrected position as an indicator of telescope wavefront. The shape of the deformable mirror is not measured directly, but is inferred from the voltages applied to its piezoelectric actuators. The actuators are known to have temperature-dependent hysteresis and is thus a source of error in this technique. In November, an interferometer was added to the $\mathrm{AO}$ system which will allow more direct measurements of corrected wavefront in future tests. Data from six measurements in July (pre-collimation) and nine measurements in September and November (post-collimation) were analyzed. The resulting phase maps show a slight difference in the wavefront with some improvement in the coma and spherical zernike terms but essentially no change (except for rotation) in astigmatism and some degradation in tricoma.
\end{abstract}

\section{Measurements performed}

The Lick AO system allows measurement of the static component of wavefront in the following manner. Prior to the cvening's observations, the AO system is calibrated using in internal point source (which is, physically, the end of a fiber optic whose mode field diameter is smaller than the diffraction-limit of the optics that follow) at HeNe wavelength. The point source is located at the Shane Telescope cassegrain focal point and feeds into the AO system. The image of the source at the AO "scoring" camera is made diffraction limited by adjusting the degrees of freedom of the deformable mirror. This adjustment takes up any aberrations introduced by the $\mathrm{AO}$ system itself and the resulting shape of the deformable mirror defines the reference zero-point for the wavefront phase. The static voltage commands that hold this mirror shape are recorded.

When the telescope is pointed to a star and the AO system turned on, the deformable mirror takes on another shape, which is time-varying due to the turbulent atmospheric component of phase, but also has a static component that is indicative of the fixed aberrations introduced by the primary-secondary. The voltages used to hold this mirror shape (averaged over several atmospheric turnover times) is recorded.

These measurements were taken during the AO runs in July, September, and November, 1998. The datasets used are summarized in Table 1.

Since the data recorded consists of a set of 61 mirror actuator voltages, it is necessary to independently measure the mirror displacement as a function of voltage on individual actuators. With the introduction of the Phase Shifting Diffraction Interferometer (PSDI) on to the AO system in November, 1998, we were able to measure phase directly, and repeated earlier calibration measurements that were done using the phase diversity method. The result is that the actuators respond with approximately $300 \mathrm{~nm}$ peak wavefront phase per Volt (150 nm mirror displacement) in a roughly Gaussian shapped influence function. The actuator gain constant varies as much as $10-15 \%$ with temperature, and is also subject to considerable hysteresis, particularly in cold weather. Figure 1 shows the difference between PSDI measurements of phase before and after pushing deformable mirror actuator number 15 . The peak is $300 \mathrm{~nm}$. Visible in the image are fringes due to internal reflections in the PSDI and shadows of the spiders in the AO pupil mask. 


\begin{tabular}{|c|c|c|c|c|}
\hline Night of & Time (PDT) & Dataset Name & Source & Mirror Temp (F) \\
\hline $7 / 4 / 98$ & $9: 39 \mathrm{pm}$ & Jul04_47 & fiber & \\
\hline $7 / 4 / 98$ & $12: 30 \mathrm{am}$ & Jul04_50 & star & N/A \\
\hline $7 / 5 / 98$ & $9: 40 \mathrm{pm}$ & Jul05_26 & fiber & \\
\hline $7 / 5 / 98$ & $12: 57 \mathrm{am}$ & Jul06_02 & HR6026 & N/A \\
\hline $7 / 5 / 98$ & 5:07 am & Jul06_19 & HD225023 & N/A \\
\hline $7 / 6 / 98$ & $9: 57 \mathrm{pm}$ & Jul06_27 & fiber & \\
\hline $7 / 6 / 98$ & $12: 18 \mathrm{am}$ & Jul06_34 & HD162208 & 69.1 \\
\hline $7 / 7 / 98$ & $8: 16 \mathrm{pm}$ & Jul07_06 & fiber & \\
\hline $7 / 7 / 98$ & $9: 52 \mathrm{pm}$ & Jul07_09 & HR5938 & 70.7 \\
\hline $7 / 8 / 98$ & $7: 30 \mathrm{pm}$ & Jul08_01 & fiber & \\
\hline $7 / 8 / 98$ & $10: 54 \mathrm{pm}$ & Jul08_07 & GL614 & 70.9 \\
\hline $9 / 7 / 98$ & $9: 14 \mathrm{pm}$ & Sep07_05 & fiber & \\
\hline $9 / 7 / 98$ & $11: 04 \mathrm{pm}$ & Sep07_15 & SAO32737 & N/A \\
\hline $9 / 13 / 98$ & $7: 29 \mathrm{pm}$ & Sep13_16 & fiber & \\
\hline $9 / 13 / 98$ & $8: 21 \mathrm{pm}$ & Sep13_19 & GL614 & N/A \\
\hline $11 / 7 / 98$ & 3:55 am & Nov07_09 & fiber & \\
\hline $11 / 7 / 98$ & $12: 47 \mathrm{am}$ & Nov07_07 & HR999 & 40.9 \\
\hline $11 / 11 / 98$ & $9: 10 \mathrm{pm}$ & Nov11_03 & fiber & \\
\hline $11 / 11 / 98$ & 1:09 am & Nov11_14 & HR1419 & N/A \\
\hline $11 / 13 / 98$ & $4: 29 \mathrm{pm}$ & Nov13_28 & fiber & \\
\hline $11 / 13 / 98$ & $10: 20 \mathrm{pm}$ & Nov13_32 & 59 And & N/A \\
\hline $11 / 13 / 98$ & $10: 22 \mathrm{pm}$ & Nov13_33 & 59 And & N/A \\
\hline $11 / 13 / 98$ & $2: 40 \mathrm{am}$ & Nov13_57 & SAO 96473 & N/A \\
\hline $11 / 13 / 98$ & $3: 52 \mathrm{am}$ & Nov13_60 & SAO 96473 & N/A \\
\hline $11 / 14 / 98$ & $11: 00 \mathrm{pm}$ & Nov14_04 & fiber & \\
\hline $11 / 14 / 98$ & 3:04 am & Nov15_10 & SAO 13788 & N/A \\
\hline
\end{tabular}

Table 1 - Measurement datasets

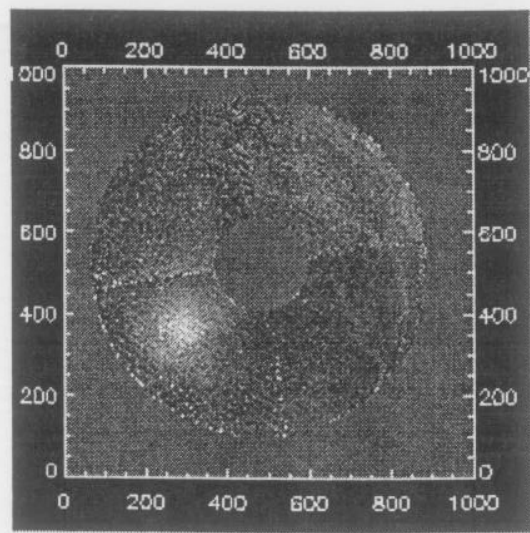

Figure 1. Phase map of deformable mirror with actuator \#15 pushed $150 \mathrm{~nm}$ (1 volt command to the PZT high voltage amplifier), subtracted from a similar map with all actuators at zero volts. 


\section{Analysis and results}

The analysis consists of reproducing the phase map given the actuator voltages for calibration and star sources, and taking the difference. This is followed by fitting the individual phase maps to Zernike basis functions. Statistics on the Zernike coefficients were calculated over the ensemble of measurements. Preand post-collimation Zernike coefficient statistics are summarized in Table 2. The Zernike basis functions are normalized to have an rms value of unity, so the coefficients have units of nanometers rms.

Phase maps from individual measurements are shown in Figures $2 \mathrm{a}$ and $2 \mathrm{~b}$. In the post-collimation data, two datasets from November 13, using star SA096473, appeared to be outliers, so the statistical data analysis was repeated with this data removed. This resulted in only subtle changes in average Zernike values and in the appearance of the average phase map. Retaining or removing this data would not affect the conclusions.

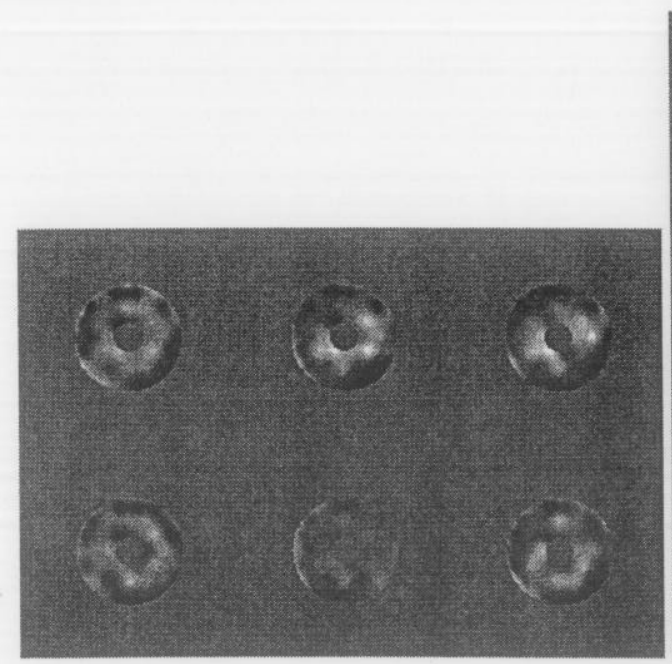

A

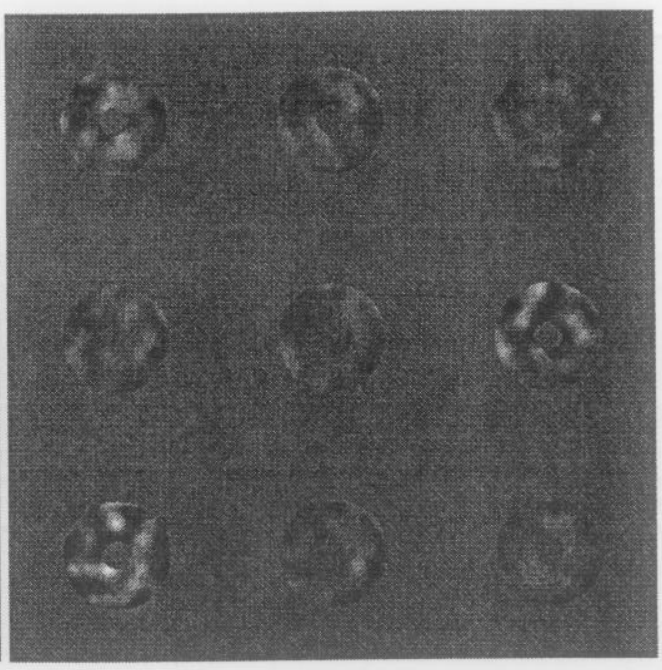

B

Figure 2. Phase maps from individual data files. A) Six phase maps taken during the July run (precollimation). B) Nine phase maps taken during September and November runs (post-collimation)

Table 2. Statistics of Zernike decomposition of phase maps. Zernikes 0-3 which correspond to piston, tip, tilt, and focus, are ignored in the analysis.

\begin{tabular}{c|cccc}
\hline Zernike \# & $\begin{array}{l}\text { Pre-collimation } \\
\text { average } \\
\text { coefficient }\end{array}$ & $\begin{array}{l}\text { Pre-collimation } \\
\text { coefficient } \\
\text { standard deviation }\end{array}$ & $\begin{array}{l}\text { Post-collimation } \\
\text { average } \\
\text { coefficient }\end{array}$ & $\begin{array}{l}\text { Post-collimation } \\
\text { standard } \\
\text { deviation }\end{array}$ \\
\hline 4 & 53.6 & 40.6 & 67.3 & 25.3 \\
5 & 46.2 & 22.9 & -20.7 & 31.0 \\
6 & 30.1 & 17.6 & 31.1 & 33.9 \\
7 & -30.7 & 16.3 & -35.9 & 18.0 \\
8 & -5.5 & 12.5 & -23.5 & 15.0 \\
9 & 13.4 & 17.7 & -4.7 & 19.5 \\
10 & 11.4 & 15.1 & -11.2 & 26.9 \\
11 & -7.5 & 18.0 & 11.6 & 32.0 \\
12 & -2.8 & 15.8 & -3.8 & 17.2 \\
13 & -9.5 & 17.5 & 14.0 & 18.4 \\
14 & -3.0 & 13.7 & 17.0 & 22.8 \\
15 & -18.5 & 3.6 & 10.3 & 19.3 \\
16 & -7.9 & 11.9 & 1.3 & 19.5 \\
17 & 1.1 & 16.9 & 7.9 & 26.6 \\
18 & -1.4 & 11.4 & -0.4 & 26.2
\end{tabular}




\begin{tabular}{l|rrrr}
19 & 5.4 & 7.9 & -22.5 & 17.5 \\
20 & -5.7 & 7.0 & -13.7 & 12.5 \\
\hline
\end{tabular}

The average pre- and post-collimation phase maps are shown in Figure 3. Figure 4 shows these same phase maps filtered with Zernike bases functions \#4 through 10, that is, showing only the astigmatism through spherical terms. Higher order terms are most likely artifacts of assumptions on the exact shape of deformable mirror actuator influence functions and/or can be caused by random drifts in the actuator gain coefficient, so the filtered maps more clearly show the affects of collimation. Zernike basis functions are shown in Figure 5 for reference.

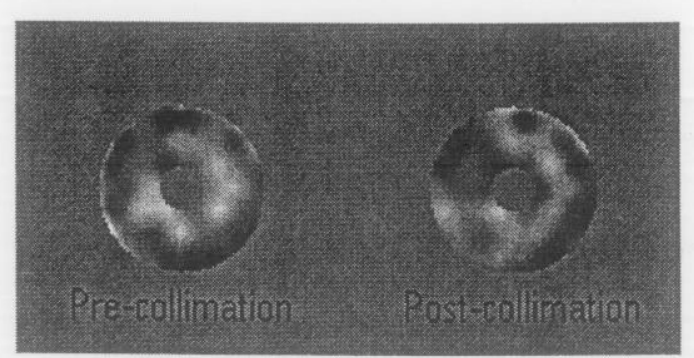

Figure 3. Average phase maps

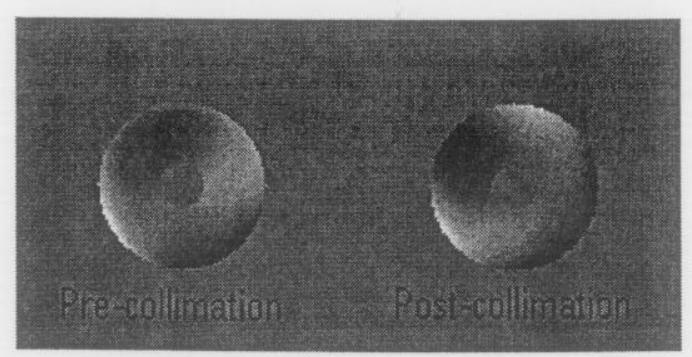

Figure 4. Average phase maps, filtered through Zernikes \#4-10

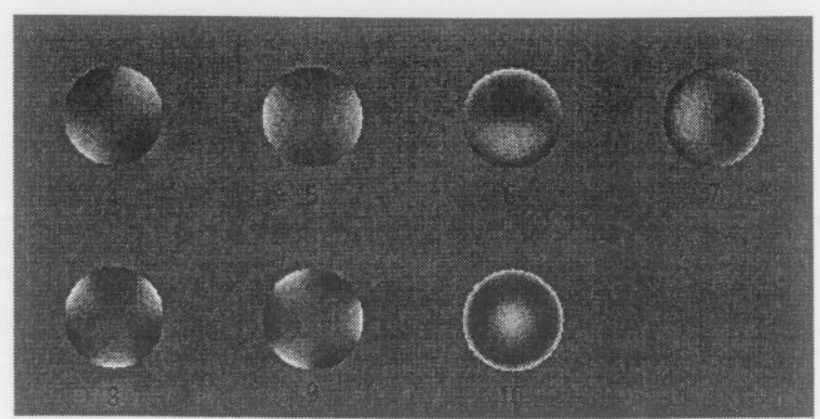

Figure 5. Zernike basis functions \#4-10 
Point spread functions corresponding to the pre- and post-collimation average, Zernike filtered, phase maps are shown in figure 6 . The pre-collimation image clearly shows coma-like tendencies, whereas the postcollimation image seems to be more rotationally symmetric, although not obviously any better in terms of compactness.
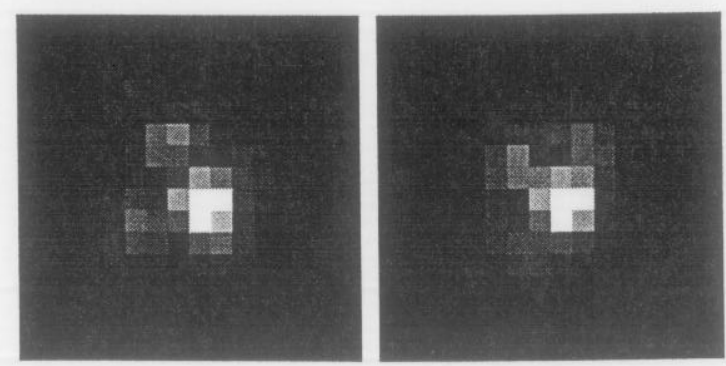

Figure 6. Pre and Post-collimation point spread functions corresponding to phase maps in Figure 5.

Wavelength is $633 \mathrm{~nm}$. One pixel corresponds to 0.02 arcseconds.

\section{Conclusions}

From Table 2, we see that astigmatism (Zernikes 4 and 5), although "rotated" is not reduced significantly after collimation. Zernike coma 6 is reduced by half while coma term 7 is essentially unchanged. The tricoma terms ( 8 and 9$)$ are somewhat interchanged but the rss of the post-collimation terms is somewhat higher than for pre-collimation. Spherical term 10 is reduced considerably after collimation. The rss error for Zernikes 4 through 10 in the post-collimation map is $98 \mathrm{~nm}$ compared to $88 \mathrm{~nm}$ rms in the precollimation map. So, by this measure, we cannot conclude that collimation improved the wavefront. The high standard deviations in the Zernike statistics indicate the difficulty in getting consistent measurements.

Using the $\mathrm{AO}$ system to measure telescope aberration currently relys on an indirect and error prone process which involves incorporating a deformable mirror actuator response model. The model is subject to uncertainties in the influence function, time/temperature variability, and hysteresis. With the recently installed phase shifting diffraction interferometer (PSDI) we can now make measurements of the deformable mirror shape directly, without using a response model. However, the present AO bench layout limits our practical use of PSDI to calibration periods during the afternoon of a run. An optical layout change scheduled to take place this year will make it feasible to do regular PSDI measurements while tracking stars, at which time we can make more reliable measurements of the telescope aberration. 